\title{
BMJ Open Study protocol for evaluating the effectiveness of depression management on gylcaemic control in non- communicable diseases clinics in Malawi
}

\author{
Michael Mphatso Udedi, ${ }^{1,2,3}$ Brian W Pence, ${ }^{4}$ Felix Kauye, ${ }^{1,3}$ Adamson S Muula ${ }^{3,5}$
}

To cite: Udedi MM, Pence BW, Kauye $\mathrm{F}$, et al. Study protocol for evaluating the effectiveness of depression management on gylcaemic control in noncommunicable diseases clinics in Malawi. BMJ Open 2018;8:e021601. doi:10.1136/ bmjopen-2018-021601

- Prepublication history for this paper is available online. To view these files please visit the journal online (http://dx.doi org/10.1136/bmjopen-2018021601).

Received 10 January 2018 Revised 18 May 2018

Accepted 5 September 2018

Check for updates

(c) Author(s) (or their employer(s)) 2018. Re-use permitted under CC BY.

Published by BMJ.

${ }^{1}$ Department of Mental Health, College of Medicine, University of Malawi, Blantyre, Malawi

${ }^{2}$ Department of Clinical Services, Ministry of Health, Lilongwe,

Malawi

${ }^{3}$ Department of Public Health, School of Public Health and Family Medicine, College of Medicine, University of Malawi, Blantyre, Malawi

${ }^{4}$ Department of Epidemiology, University of North Carolina at Chapel Hill, Chapel Hill, North Carolina, USA

${ }^{5}$ Africa Center of Excellence in Public Health and Herbal Medicine, College of Medicine, University of Malawi, Blantyre, Malawi

Correspondence to Michael Mphatso Udedi; mphatsoudedi@yahoo.co.uk

\section{ABSTRACT}

Introduction Depression is associated with negative patient outcomes for chronic diseases and likely affects consistent physical non-communicable diseases (NCDs) care management in relation to clinic attendance and medication adherence. We found no published studies on the integration of depression management in physical NCD clinics in Malawi and assessing its effects on patient and service outcomes. Therefore, the aim of this study is to evaluate the effectiveness of integrating depression screening and management in physical NCD routine care on patient and service outcomes in Malawi. We will also determine the sensitivity and specificity of the Patient Health Questionnaire-9 (PHQ-9) in the detection of depression in NCD clinics.

Methods and analysis The study will have two phases. Phase I will involve the validation of the PHQ-9 screening tool for depression, using a cross-sectional study design involving 323 participants, in two specialised physical NCD clinics in one of the 28 districts of Malawi. Using a quasi-experimental study design in four districts of Malawi not involved in the phase I study, the phase II study will evaluate the effectiveness of integrating depression screening (using $\mathrm{PHQ}-9$ ) and management (based on a specially designed toolkit). Outcomes will be measured at 3 months and 6 months among patients with comorbid diabetes (poorly controlled) and depression attending physical NCD clinics in Malawi.

Ethics and dissemination Ethical approval was obtained from the University of Malawi, College of Medicine Research and Ethics Committee (COMREC) on 31 August 2017 (reference P.07/17/2218). The findings will be disseminated through presentations at journal clubs, senior management of the Ministry of Health, national and international conferences as well as submission to peerreviewed publications. Policy briefs will also be created. Trial registration number PACTR201807135104799.

\section{INTRODUCTION}

Physical non-communicable diseases (NCDs) and depression individually are common and significant causes of morbidity, disability and mortality in sub-Saharan Africa. ${ }^{1}$ NCDs account for a growing burden on the population and health systems in
Strengths and limitations of this study

- The cross-sectional study design in phase I will allow us to estimate the prevalence of depression among people with diabetes.

- The quasi-experimental design will enable us to investigate the impact of depression in determining the outcome of diabetes.

- The quasi-experimental design which will be used in the second phase has a limitation that baseline differences between the clinics may lead to spurious results.

low-income and middle-income countries (LMICs). ${ }^{2}$ Similarly depression is projected to become a leading cause of disability by $2030 .^{3}$ The World Health Survey of 2007 suggests that the prevalence of comorbidity of physical NCDs and depression is increasing; thus between $9.3 \%$ and $23.0 \%$ of people with NCDs had comorbid depression worldwide. ${ }^{4}$ The global prevalence of major depression in people with diabetes is $27 \% .^{5}$ The relationship between depression and physical NCDs is bidirectional. Modifiable risk factors for physical NCDs are exacerbated by poor mental health and on the other hand physical NCDs are a risk factor for mental illness. ${ }^{6}$ Comorbidity is associated with a range of outcomes, including poor adherence or non-adherence to medication, functional impairment, increased medical costs, poor self-care regimens, increased medical symptom burden, and increased morbidity and mortality. ${ }^{7-9}$ The recent international study involving 14 countries shows that people with diabetes have depressive disorder as well as significant levels of depressive symptoms. Management of depression (pharmacological treatment combined with psychotherapy) in diabetes mellitus has been shown to be effective both on depression outcomes as well as diabetes management and glycaemic control. ${ }^{1011}$ 
Physical NCDs are common and in Malawi, it is estimated that NCDs account for at least $12 \%$ of total disability-adjusted life years. ${ }^{12}$ In addition, NCDs are the second leading cause of deaths in adults after AIDS. ${ }^{12}$ Furthermore, a 2009 nationwide STEPwise approach to Surveillance Survey showed that about $6 \%$ of the general population had diabetes mellitus. ${ }^{13}$

Depression commonly overlaps with physical NCDs and depression complicates NCD care. We are not aware of any studies done in Malawi that assessed the burden of depression in physical NCD care and its effect on diabetes outcomes. A recent study done in one of the 28 districts of Malawi reported that control of diabetes is poor and there was high non-adherence of NCD clients to appointments. ${ }^{14}$ Currently there are no published studies done in Malawi to explore factors associated with poor control of diabetes and the high rate of non-adherence to attending appointments in physical NCD clinics.

In Malawi, there are few studies looking at depression screening and treatment in general care. The prevalence of depression among outpatient attendees in primary care clinics in Malawi in 2010 and 2013 ranged between $19 \%$ to $30 \%{ }^{15}{ }^{16}$ Despite the high prevalence of depression in general healthcare services in Malawi, the extent of comorbid depression in the context of NCDs is unclear. Using the results from general primary care clinic studies done in Malawi on detection rates for depression, it is likely that detection rates of depression in physical NCD clinics in Malawi are poor. Undiagnosed and untreated depression more likely contributes to poor control of diabetes as shown in studies done in other countries. ${ }^{17-20}$ Evidence from a recent international study shows that identification and treatment of mental health problems is not routine in clinical care. ${ }^{20}$ In Malawi, routine screening will assist in early detection of patients with comorbid depression in physical NCD clinics and facilitate treatment. To our knowledge, there are no studies done in Malawi focusing on identification and interventions for depression in physical NCD clinics. The integration of mental healthcare into other health services is one of the strategies in the National Mental Health Policy in Malawi. ${ }^{21}$ The results of the proposed study will help to inform on decisions whether to integrate the strategies for depression management in physical NCD clinics in Malawi.

\section{AIMS AND OBJECTIVES}

Our overall objective is to evaluate the effectiveness of integrating screening and depression management into physical NCD routine care on patient and service outcomes in Malawi. In this way, we will contribute to evidence on comorbidity of depression and diabetes including the effects of identifying and treating depression in patients with comorbid diabetes and depression in physical NCD care in Malawi.

Our study has four specific objectives:
1. To determine the sensitivity and specificity of the Patient Health Questionnaire-9 (PHQ-9) in the detection of depression in NCD clinics.

2. To estimate the prevalence of comorbidity between depression and diabetes mellitus in Malawi.

3. To determine detection rate of depression in physical NCD clinics in Malawi.

4. To compare patient outcomes in physical NCD clinics with and without integration of depression screening and management.

\section{RESEARCH QUESTIONS}

The main research questions in this study are:

1. Will the validated Chichewa PHQ-9 demonstrate satisfactory sensitivity and specificity in physical NCD clinic settings in Malawi?

2. Will integrating depression screening using a validated PHQ-9 and depression management (based on a specially designed toolkit) be more effective than usual care in identifying patients with comorbid depression and diabetes, facilitating treatment of depression in patients with comorbid depression and diabetes, and improving clinical outcomes among patients with comorbid depression and diabetes?

\section{METHODS AND ANALYSIS}

The study will be conducted from December 2017 to December 2018. The study will be in two phases. Phase I will be for validation of PHQ-9 among diabetes patients. Phase II will be evaluating the effectiveness of integrating screening and depression management in patients with comorbid depression and diabetes attending physical NCD clinics. To answer the research questions, we will use a cross-sectional study design in phase I and a quasi-experimental study design in phase II.

\section{Patient and public involvement}

Patients and or public were not involved in the design of this study; however the results will be disseminated to study participants through the clinics' diabetes support groups.

\section{Phase I: Validation of PHQ-9 in a physical NCD clinic setting in Malawi}

Study design, area and physical NCD clinics

Depression varies based on cultural context as such screening tools must be adapted and validated for particular populations. PHQ-9 has not been validated in Malawi hence the study will assess validity of PHQ-9. For phase I, we will use a cross-sectional study design. The study will be conducted in Lilongwe district which is one of the 28 districts in Malawi. Malawi is divided administratively into three regions namely Northern, Central and Southern. Lilongwe is located in the central region of Malawi. It has a population of 2688379 people $^{22}$ with eight physical NCD clinics. 


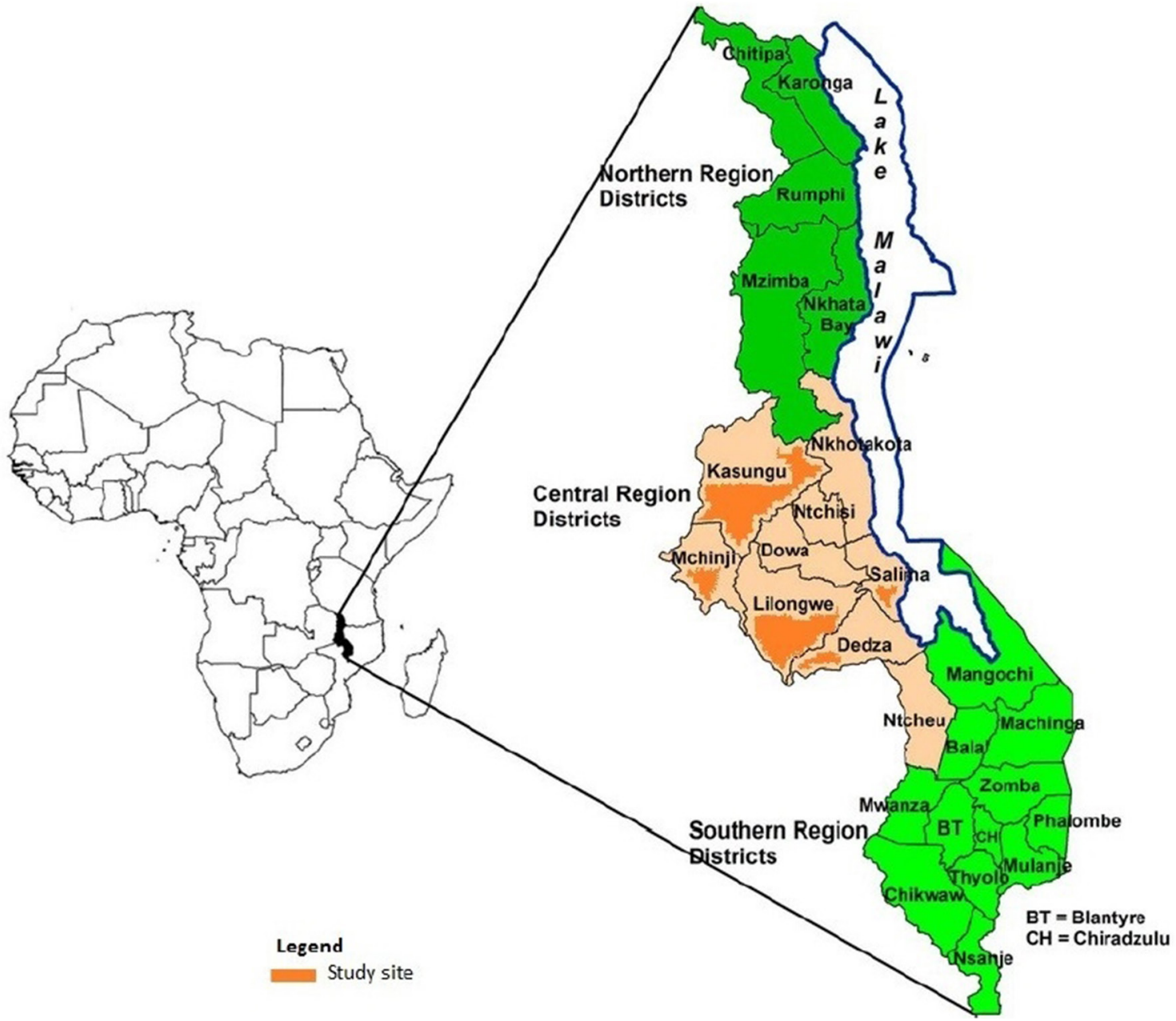

Figure 1 Map of Malawi showing the central region districts and study sites.

The study will be conducted in two physical NCD clinics (Area 25 Health Centre under Lilongwe District Health Office and Kamuzu Central Hospital). The Area 25 Health Centre serves a catchment population of 80 000 while the catchment population of Kamuzu Central Hospital (a tertiary health facility) is the entire central region which is $7401068 .^{22}$ The physical NCD clinic at Area 25 Health Centre is staffed by clinical officers and nurses who have undergone appropriate training in the diagnosis and management of key NCDs while the clinic of Kamuzu Central Hospital is also staffed by medical officers (general medical doctors) and specialists on top of clinical officers and nurses. The two sites are implementing the WHO Package of Essential NCD (WHO PEN) interventions. Figure 1 is a map of Malawi showing the central region districts.

\section{SAMPLE SIZE DETERMINATION}

The sample size was calculated based on the sensitivity of PHQ-9 using Buderer's formula. ${ }^{23}$ We used the following parameters; anticipated sensitivity was $80 \%$, standard normal deviation corresponding to the specified size of the critical region $\left(\mathrm{z}^{2}{ }_{1-}{ }_{\alpha / 2}\right)$ is 3.84 , the size of the critical region, alpha $(\alpha)$, is $0.05, \mathrm{z}$ is corresponding to the level of significance and is $1.96, \mathrm{~L}$ is the absolute precision desired on either side of sensitivity and was set at 0.1 and prevalence for depression was $20 \%$. The prevalence of depression of $20 \%$ is based on a recent study on patients attending a healthcare setting in Malawi. ${ }^{15}$ We also considered potential participants' refusal and loss of data; consequently we put it at 5\%. Taking these assumptions into consideration, the required sample size was calculated as 323 .

\section{RECRUITMENT AND DATA COLLECTION}

Consecutive patients attending the NCD clinic will be identified by research assistants. The research assistants will provide information about the study to the patients and seek informed written consent. Participants will be eligible for the study if they are at least 18 years of age and are attending the NCD clinics for diabetes care and are available for an interview. Participants will be excluded if they require acute medical care or are unable to speak.

The research assistants will enrol patients who consent and carry out interviews at the health facility. When patients do not consent to participate in the study, the research assistant will request permission to record sex, age, level of education and occupation of the patient. 


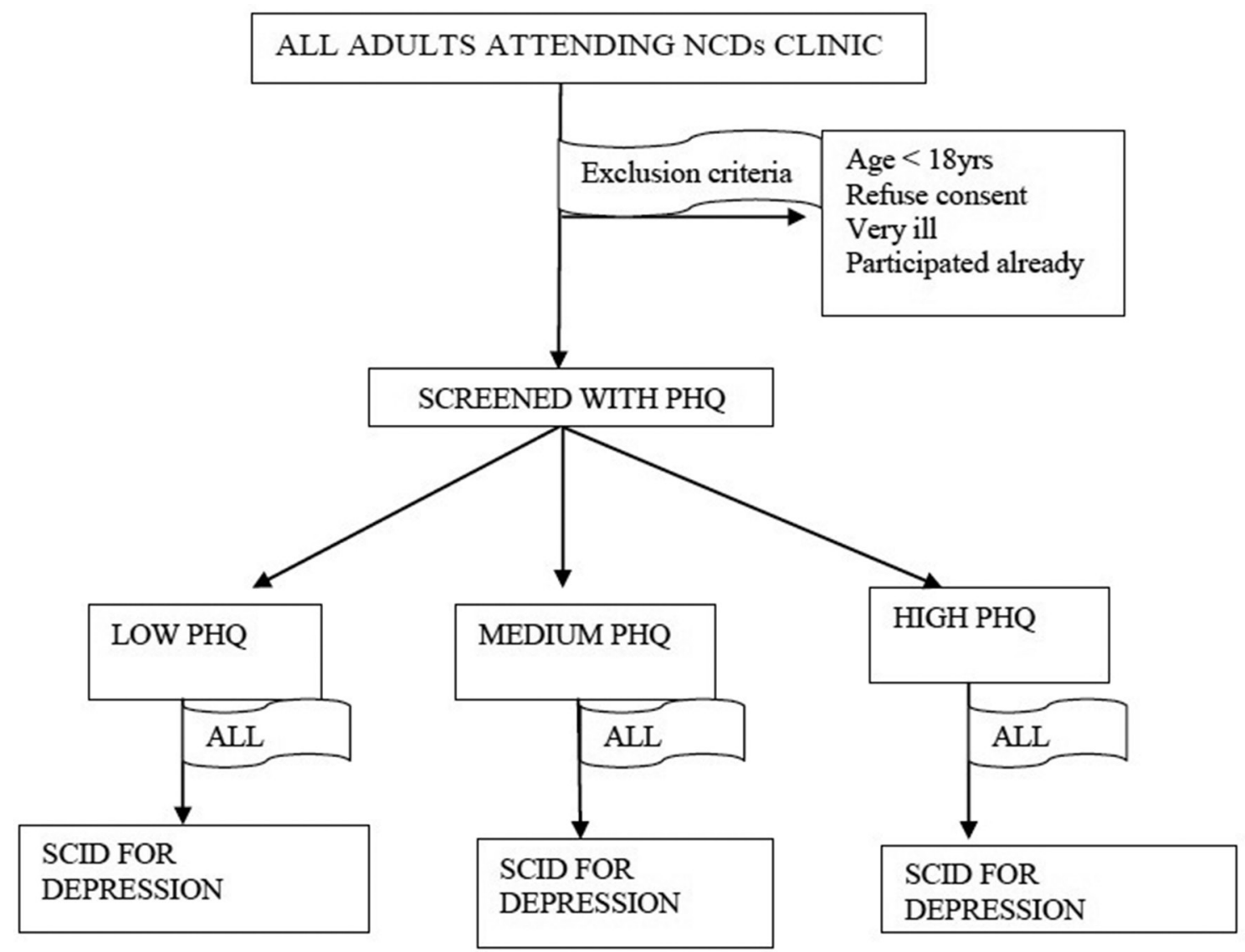

Figure 2 Phase I study procedure. NCD, non-communicable disease; PHQ, Patient Health Questionnaire.

One research assistant will administer PHQ-9 orally to ensure high and standard responses due to low literacy of the study participants. The research assistant will then calculate the screening score and let the participant be attended to by the NCD clinician. After the consultation the patient will have the Diagnostic and Statistical Manual of Mental Disorders (DSM-IV), Axis I disorders Clinical Version Structured Clinical Interview for DSM (SCID) administered by a second research assistant to elicit the psychiatric symptoms itemised in the DSM criteria for depression. The second research assistant will be masked from the PHQ-9 Score. The training, supervision and quality assurance of the research assistant doing the SCID will be done as well. A diagrammatic representation of the process is shown in figure 2 .

\section{MEASUREMENTS}

The aim of phase I is to validate PHQ-9 in physical NCD clinics in order for it to be used for screening of depression in NCDs in Malawi. PHQ-9 (based on DSM criteria) has been chosen for this study because it is brief, requires a short time to administer and is compatible with the Malawian health setting workload. Furthermore, the PHQ-9 can be used to establish depressive disorder diagnoses as well as depressive symptoms' severity. ${ }^{24}$

For screening data using PHQ-9, presence of depressive symptoms will be defined as having a total score of 5 and above on all the nine questions (PHQ-9 total score $\geq 5$ ). Symptoms will be categorised using established cut-off points 5, 10 and 20 (mild, moderate and severe depressive symptoms, respectively). ${ }^{25} 26$ The sensitivity and specificity at the standard cut-off point will be assessed, and performance at an alternative cut-off point will also be examined to determine whether the standard cut-off point is optimal in this population

SCID for depression will be used as the gold standard to validate PHQ-9. SCID for depression has been translated into Chichewa and has been used in Malawi after undergoing a process of validation which included translation, back translation and testing. ${ }^{27}$

\section{Data management}

Data will be checked for consistency and completeness by the principal investigator and double entered to SPSS V.20 by experienced data entry clerks. Then, it will be cleaned and analysed using the same V.20 of SPSS. Hard copies of the data will be stored in a locked cabinet and consent forms will be separated from the data.

\section{Data analysis}

Data will be analysed using SPSS V.20. Descriptive statistics will be computed to describe the sociodemographic characteristics of participants and to summarise the frequencies of depression under each category in both criteria (PHQ-9 and SCID). We will assess whether refusal 
is associated with sociodemographic characteristics by testing the statistical significance of sociodemographic characteristics between refusers and consenters. The prevalence of diagnosed depression among patients with diabetes will be determined by computing the proportion of those with depression according to SCID; these patients will be correctly screened out by PHQ.

The sensitivity, specificity and predictive values of a PHQ-9 total score $\geq 5$, with $95 \%$ CIs, will be calculated relative to the reference standard of a diagnosis from the depression module of SCID. A receiver operating characteristic curve (ROC curve) will be made using SPSS V.20 in order to get criterion validity of PHQ-9. Internal consistency will be determined by Cronbach's $\alpha$. In exploratory analyses we will examine the test characteristics of alternative thresholds.

\section{Phase II: evaluation of the effectiveness of integrating} screening using validated $\mathrm{PHQ}-9$ and depression management in physical NCD clinics

Study design and setting

In phase II of the study, we will use a quasi-experimental design employing a non-equivalent control group (prepost) design. Prospective controlled interventional trials provide a higher level of evidence for a true causality. ${ }^{28}$ The best choice of the controlled interventional studies is the true experimental design of which RCT is the gold standard. However a non-randomised control group trial is a reasonable choice when an RCT would be ideal but not feasible due to costs and ethics limitations and also when trying to establish the effectiveness of large-scale dissemination and implementation. ${ }^{28} 29$ Furthermore, literature indicates that the interventions often cannot be randomised for the following reasons: ethical considerations, an inability to randomise patients, an inability to randomise locations or settings, and a need to intervene quickly. ${ }^{29}$ We have therefore chosen to use a quasi-experimental design and the intervention programme will be done in two district NCD clinics while the other two districts will provide usual care. Four convenient physical NCD clinics which are implementing the WHO PEN interventions will be allocated to intervention arm (two physical NCD clinics) and control arm (two physical NCD clinics) and in each clinic. Consecutive patients who meet the study criteria will be recruited.

The phase II of the study will be conducted for a period of 9-12 months in four healthcare facilities in Mchinji district, Kasungu district, Salima district and Dedza district of the central region of Malawi (figure 1). The four districts are also implementing the WHO PEN interventions. Currently each district has one physical NCD clinic based at the district hospital. The physical NCD clinics in the four districts are staffed by clinical officers and nurses who have undergone appropriate training in the diagnosis and management of key physical NCDs. They follow similar standard guidelines in the management of physical NCDs.

\section{Intervention}

1. Clinicians at the physical NCD clinics will be trained in routine screening for depression with a validated PHQ-9.

2. Clinicians at the physical NCD clinics will be trained on comorbidity of diabetes and depression and on coming up with individual care plans and prescribing and monitoring antidepressants.

3. Clinicians at the physical NCD clinics will be trained on problem-solving skills using effective helping techniques for depression and on how to follow-up individual care plans.

\section{Usual care}

This would involve the NCD clinicians treating patients with comorbid depression and diabetes using normal practices of treating depression involving antidepressants.

\section{SAMPLE SIZE DETERMINATION}

The sample size was based on detecting a difference in glycated haemoglobin $\left(\mathrm{HbA}_{1} \mathrm{c}\right)$ at 6 months between the study arms. It was based on an analysis of covariance (ANCOVA) approach, with the outcome measurement at follow-up, and the baseline $\mathrm{HbA}_{1} \mathrm{c}$ considered as a covariate in the analysis. The correlation between baseline and follow-up haemoglobin values is estimated to be 0.5 . A difference in $\mathrm{HbA}_{1} \mathrm{c}$ between arms of $0.7 \%$ is considered to be a clinically important difference (based on a previous study which found a difference of $0.56 \%,{ }^{30}$ ) and data from previous research suggested that the $\mathrm{HbA}_{1} \mathrm{c}$ measurements had an SD of $1.8 \% .{ }^{30}$ It is calculated that using a $5 \%$ significance level and $80 \%$ power; 78 subjects per group are required. It is estimated that there will be a $10 \%$ dropout/loss to follow-up. To allow for this, we planned to recruit 87 subjects per group, giving a total sample size for the study of 174 participants.

\section{RECRUITMENT AND DATA COLLECTION}

We will use a mixed-methods (quantitative and qualitative) approach in phase II. The reason for collecting both quantitative and qualitative data is to complement and also to address the shortfalls of either approach. The use of both forms of data allows researchers to gain deeper understanding of the phenomenon under study. ${ }^{31}$ In addition, evidence suggests that triangulation of methods increases both the validity and reliability of data. ${ }^{32}$

The research assistant with the help of health professionals running the NCD clinic at each site will identify the study participants who meet the inclusion criteria consecutively. The research assistants will provide information about the study to the patient and seek written informed consent. Participants will be eligible for the study if they are at least 18 years of age, are attending the NCD clinic for diabetes care and are available for an interview. Participants will be excluded if they require acute medical care or are unable to speak. The research 


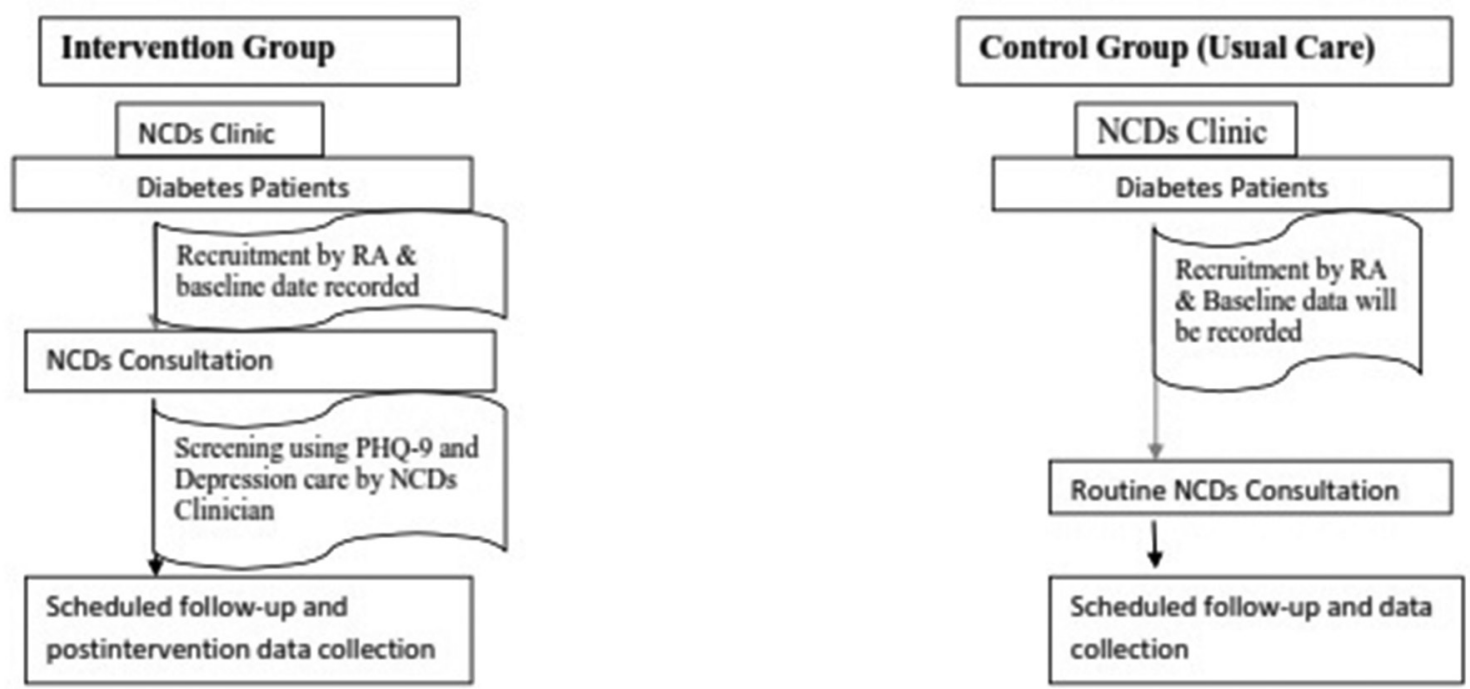

\begin{tabular}{|l|l|l|}
\hline Three months follow-up & Three months follow-up \\
\hline PHQ 9 score & & PHQ 9 score \\
\hline Diabetes control check & & Diabetes control check \\
\hline EQ-5D & & EQ-5D \\
\hline Six months follow-up & Six months follow-up \\
\hline PHQ 9 & PHQ 9 \\
\hline Diabetes control check & & Diabetes control check \\
\hline EQ-5D & & EQ-5D \\
\hline $\begin{array}{l}\text { Baseline assessments will be } \\
\text { done for all four measures \& } \\
\text { recorded }\end{array}$ & $\begin{array}{l}\text { Baseline assessments will be } \\
\text { done for three measures only } \\
\text { \& recorded }\end{array}$ \\
\hline
\end{tabular}

Figure 3 Phase II study procedure. EQ-5D, EuroQol Five Dimensions Questionnaire; NCD, non-communicable disease; PHQ9, Patient Health Questionnaire -9.

assistants will enrol patients who consent and carry out interviews at the health facility. When patients do not consent to participate in the study, the research assistant will request permission to record sex, age, level of education and occupation of the patient. Adult patients with poorly controlled diabetes $\left(\mathrm{a} \mathrm{HbA}_{1} \mathrm{c}\right.$ level of $8.5 \%$ or higher as suggested from previous research ${ }^{30} 33$ ) and comorbid depression will be recruited for the study and followed up to 6 months after recruitment (figure 3). Data collection will occur at three time points: baseline, at 3 months and at 6 months.

Qualitative interviews will be conducted among healthcare workers in order to assess feasibility and acceptability of integrating depression management into NCD care. In-depth interviews will be employed to understand health professionals' experience in using the validated PHQ-9 for screening and the depression management guidelines.

\section{MEASUREMENTS}

The primary exposure variable

Depression will be measured using PHQ-9. PHQ-9 will have been validated in phase I of this study.

The primary exposure of interest is whether the patient is receiving care at an intervention or usual care facility.

\section{Outcome variables}

Primary outcome variable

The patient diabetes outcome for this study is blood glucose control. Data on the patients' status on the primary outcome will be extracted from the NCD patient mastercard. For the purpose of the study in checking how well diabetes is being controlled, $\mathrm{HbA}_{1} \mathrm{c}$ test will be performed on the recruited study participants at baseline, 3 months and 6 months. Depression is the co-primary outcome. PHQ-9 will be administered at baseline, 3 months and 6 months. 


\section{Secondary outcome variable}

Quality of life (QoL) will be measured using the interviewer-administered version of the EuroQol Five Dimensions (EQ-5D) Questionnaire. This instrument has been validated in South Africa and Zimbabwe. ${ }^{34} 35$ The tool has been translated into several languages including Afrikaners, Xhosa and Shona. ${ }^{35}$ The EQ-5D Questionnaire will be adapted in Malawi and will be used at baseline and follow-up of patients at 3 months and 6 months.

\section{Data management}

Data will be checked for consistency and completeness by the principal investigator and double entered to SPSS V.20 by experienced data entry clerks. Hard copies of the data will be stored in a locked cabinet and consent forms will be separated from the data.

\section{Data analysis}

The primary outcome is $\mathrm{HbA}_{1} \mathrm{c}$ at 6 months. This will be compared between groups using ANCOVA. The haemoglobin value at baseline will be considered as a covariate in the analysis.

The $\mathrm{HbA}_{1} \mathrm{c}$ values at 3 months will be considered as a separate outcome, and analysed using equivalent methods to the primary outcome.

Quantitative data will be analysed statistically using SPSS V.20. Descriptive analysis will be done. The recorded qualitative data on the feasibility and acceptability of integrating depression management will be transcribed and coded using Atlas.ti. Relevant queries will be created in the database to thematically analyse the data.

\section{Ethical considerations}

Written informed consent will be obtained from every participant and fingerprint impressions will be taken from consenting illiterate participants. Patients identified as having depression or endorsing the suicide item on PHQ-9 will be referred to appropriate professionals for further assessment and management. Permission to conduct the study has been sought from the the district health officers of Lilongwe, Kasungu, Dedza, Mchinji, Salima and the hospital director of Kamuzu Central Hospital. Study participants will not be given any financial reimbursement, however they will thanked verbally for their participation. Privacy will be maintained as the interviews will be conducted in a room which will provide both visual and audio privacy. Referrals will be made to appropriate professionals for further assessment and management of cases where participants will be identified to be under depression as well as cases that will screen positive for suicidal ideations through the interviews.

\section{Dissemination plan}

The findings will be disseminated through publications in peer-reviewed journals and conference presentations. Summary reports will be submitted to the participating institutions and to the University of Malawi, College of Medicine. The results will also be shared with policy makers and other local stakeholders in the form of policy briefs as part of evidence on integration of depression management in NCDs.

\section{Limitations of the study}

In this study, people may have undiagnosed comorbid illnesses and our method of screening may not be strong in capturing other comorbid illnesses. In the first phase of validation we will use two specialised clinics which may not be representative of the wider population. QoL will be measured using five domains and we will therefore not obtain detailed information on the various dimensions that assess health-related QoL. The quasi-experimental design which will be used in the second phase also has a limitation that baseline differences between the clinics may lead to spurious results.

\section{Strengths of the study}

This study will provide the much needed evidence about the impact of comorbid depression on the outcome of diabetes. The cross-sectional study design will allow us to estimate the prevalence of depression among people with diabetes. The study will enable us to investigate the impact of depression in determining the outcome of diabetes. To our knowledge, this study will be the first in Malawi to examine identification and interventions for depression in physical NCD clinics.

\section{Expected benefits of the findings}

Our findings will contribute to sparse evidence on mental health, diabetes and other NCDs in LMICs. We anticipate that our study findings will raise awareness of comorbidity among clinicians, service providers, policy makers and that it will contribute to clinical practice and policy change regarding the delivery of mental healthcare in NCD care in Malawi.

Contributors MMU, BWP, FK and ASM were involved in the conceptualisation of the study. MMU was responsible for drafting the protocol manuscript. BWP, FK and ASM edited the protocol. All authors read and approved the final manuscript.

Funding This study protocol presents independent work supported through the DELTAS Africa Initiative (DEL-15-01). The DELTAS Africa Initiative is an independent funding scheme of the African Academy of Sciences' (AAS) Alliance for Accelerating Excellence in Science in Africa (AESA) and supported by the New Partnership for Africa's Development Planning and Coordinating Agency (NEPAD Agency) with funding from the Wellcome Trust (DEL-15-01) and the UK government.

Disclaimer The views expressed in this publication are those of the author(s) and not necessarily those of AAS, NEPAD Agency, Wellcome Trust or the UK government. Competing interests None declared.

Patient consent Not required.

Ethics approval University of Malawi, College of Medicine Research and Ethics Committee (COMREC)

Provenance and peer review Not commissioned; externally peer reviewed.

Data sharing statement The authors will share data of the original research article with their colleagues for academic purposes only. Currently the authors have not shared any unpublished data for the study.

Open access This is an open access article distributed in accordance with the Creative Commons Attribution 4.0 Unported (CC BY 4.0) license, which permits others to copy, redistribute, remix, transform and build upon this work for any purpose, provided the original work is properly cited, a link to the licence is given, and indication of whether changes were made. See: https://creativecommons.org/ licenses/by/4.0/. 


\section{REFERENCES}

1. Nikolic IA, Stanciole AE, Zaydman M. Chronic emergency: why NCDs matter. Health, nutrition, and population discussion paper. 2011.

2. Buttorff C, Hock RS, Weiss HA, et al. Economic evaluation of a taskshifting intervention for common mental disorders in India. Bull World Health Organ 2012;90:813-21.

3. Mathers C, Fat DM, Boerma JT. The global burden of disease: 2004 update. Geneva, Switzerland: World Health Organization, 2008.

4. Moussavi S, Chatterji S, Verdes E, et al. Depression, chronic diseases, and decrements in health: results from the World Health Surveys. Lancet 2007;370:851-8.

5. World Health Organization. Investing in mental health. Geneva: World Health Organization, 2003.

6. Ngo VK, Rubinstein A, Ganju V, et al. Grand challenges: integrating mental health care into the non-communicable disease agenda. PLoS Med 2013;10:e1001443.

7. Mensah GA, Collins PY. Understanding mental health for the prevention and control of cardiovascular diseases. Glob Heart 2015;10:221-4.

8. Rieckmann N, Gerin W, Kronish IM, et al. Course of depressive symptoms and medication adherence after acute coronary syndromes: an electronic medication monitoring study. J Am Coll Cardiol 2006;48:2218-22.

9. Gehi A, Haas D, Pipkin S, et al. Depression and medication adherence in outpatients with coronary heart disease: findings from the Heart and Soul Study. Arch Intern Med 2005;165:2508-13.

10. van der Feltz-Cornelis CM, Nuyen J, Stoop C, et al. Effect of interventions for major depressive disorder and significant depressive symptoms in patients with diabetes mellitus: a systematic review and meta-analysis. Gen Hosp Psychiatry 2010;32:380-95.

11. Simon GE, Katon WJ, Lin EH, et al. Cost-effectiveness of systematic depression treatment among people with diabetes mellitus. Arch Gen Psychiatry 2007;64:65-72.

12. MoH, WHO. Malawi national STEPS survey for chronic noncommunicable diseases and their risk factors, 2010.

13. Msyamboza KP, Ngwira B, Dzowela T, et al. The burden of selected chronic non-communicable diseases and their risk factors in Malawi: nationwide STEPS survey. PLoS One 2011;6:e20316.

14. Manjomo RC, Mwagomba B, Ade S, et al. Managing and monitoring chronic non-communicable diseases in a primary health care clinic, Lilongwe, Malawi. Public Health Action 2016;6:60-5.

15. Kauye F, Jenkins $R$, Rahman A. Training primary health care workers in mental health and its impact on diagnoses of common mental disorders in primary care of a developing country, Malawi: a clusterrandomized controlled trial. Psychol Med 2014;44:657-66.

16. Udedi M. The prevalence of depression among patients and its detection by primary health care workers at Matawale Health Centre (Zomba). Malawi Med J 2014;26:34-7.

17. Ciechanowski P, Russo J, Katon W, et al. Where is the patient? The association of psychosocial factors and missed primary care appointments in patients with diabetes. Gen Hosp Psychiatry 2006;28:9-17.
18. Andreoulakis E, Hyphantis T, Kandylis D, et al. Depression in diabetes mellitus: a comprehensive review. Hippokratia 2012;16:205-14.

19. Ehb L, Rutter CM, Katon W, et al. Depression and advanced complications of diabetes. Diabetes Care 2010;33:264.

20. Lloyd CE, Nouwen A, Sartorius N, et al. Prevalence and correlates of depressive disorders in people with Type 2 diabetes: results from the International Prevalence and Treatment of Diabetes and Depression (INTERPRET-DD) study, a collaborative study carried out in 14 countries. Diabet Med 2018;35:760-9.

21. Ministry of Health and Population. National mental health policy. Lilongwe, Malawi: Ministry of Health and Population, 2001.

22. National Statistical Office (NSO). Population projections Malawi. Zomba, Malawi: National Statistical Office (NSO), 2009.

23. Buderer NM. Statistical methodology: I. Incorporating the prevalence of disease into the sample size calculation for sensitivity and specificity. Acad Emerg Med 1996;3:895-900.

24. Kroenke K, Spitzer RL, Williams JB. The PHQ-9: validity of a brief depression severity measure. J Gen Intern Med 2001;16:606-13.

25. Löwe B, Kroenke K, Herzog W, et al. Measuring depression outcome with a brief self-report instrument: sensitivity to change of the Patient Health Questionnaire (PHQ-9). J Affect Disord 2004;81:61-6.

26. Löwe B, Unützer J, Callahan CM, et al. Monitoring depression treatment outcomes with the patient health questionnaire-9. Med Care 2004;42:1194-201.

27. Stewart RC, Kauye F, Umar E, et al. Validation of a Chichewa version of the self-reporting questionnaire (SRQ) as a brief screening measure for maternal depressive disorder in Malawi, Africa. J Affect Disord 2009;112:126-34.

28. Axelrod DA, Hayward R. Nonrandomized interventional study designs (quasi-experimental designs). In: Penson DF, Wei JT, eds. Clinical research methods for surgeons. Totowa, NJ: Humana Press, 2007:63-76.

29. Harris AD, Bradham DD, Baumgarten M, et al. The use and interpretation of quasi-experimental studies in infectious diseases. Clin Infect Dis 2004;38:1586-91.

30. Katon WJ, Lin EH, Von Korff M, et al. Collaborative care for patients with depression and chronic illnesses. $N$ Engl J Med 2010;363:2611-20.

31. Creswell JW. Research design: qualitative, quantitative, and mixed methods approaches: Sage publications, 2013

32. Ajayi IO, Olumide EA, Oyediran O. Patient satisfaction with the services provided at a general outpatients' clinic, Ibadan, Oyo State, Nigeria. Afr J Med Med Sci 2005;34:133-40.

33. Wilkinson JE, Culpepper L. Associations between colorectal cancer screening and glycemic control in people with diabetes, Boston, Massachusetts, 2005-2010. Prev Chronic Dis 2011;8.

34. Jelsma J, Mhundwa K, De Weerdt W, et al. The reliability of the Shona version of the EQ-5D. Cent Afr J Med 2001;47:8-13.

35. Jelsma J, Ferguson $G$. The determinants of self-reported healthrelated quality of life in a culturally and socially diverse South African community. Bull World Health Organ 2004;82:206-12. 